\title{
CXCR4/CXCL12 Signaling and Protumor Macrophages in Primary Tumors and Sentinel Lymph Nodes Are Involved in Luminal B Breast Cancer Progression
}

\author{
Carlotta Raschioni (D), ${ }^{1}$ Giulia Bottai, ${ }^{1}$ Andrea Sagona, ${ }^{2}$ Valentina Errico, ${ }^{3}$ Alberto Testori, ${ }^{3}$ \\ Wolfgang Gatzemeier, ${ }^{2}$ Fabio Corsi $\left(10,{ }^{4,5}\right.$ Corrado Tinterri, ${ }^{2}$ Massimo Roncalli, ${ }^{6,7}$ \\ Libero Santarpia, ${ }^{1}$ and Luca Di Tommaso $\mathbb{1}^{6,7}$ \\ ${ }^{1}$ Oncology Experimental Therapeutics, Humanitas Clinical and Research Center, Rozzano, Milan, Italy \\ ${ }^{2}$ Senology Unit, Humanitas Clinical and Research Center, Rozzano, Milan, Italy \\ ${ }^{3}$ Thoracic Surgery Unit, Humanitas Clinical and Research Center, Rozzano, Milan, Italy \\ ${ }^{4}$ Laboratory of Nanomedicine, Surgery Division, Department of Biomedical and Clinical Sciences, University of Milan, \\ "Luigi Sacco" Hospital, Milan, Italy \\ ${ }^{5}$ Breast Unit, Surgery Division, ICS Maugeri, Pavia, Italy \\ ${ }^{6}$ Pathology Unit, Humanitas Clinical and Research Center, Rozzano, Milan, Italy \\ ${ }^{7}$ Department of Biomedical Sciences, Humanitas University, Rozzano, Milan, Italy
}

Correspondence should be addressed to Luca Di Tommaso; luca.di_tommaso@humanitas.it

Received 28 September 2017; Accepted 17 January 2018; Published 16 April 2018

Academic Editor: Alvaro González

Copyright (c) 2018 Carlotta Raschioni et al. This is an open access article distributed under the Creative Commons Attribution License, which permits unrestricted use, distribution, and reproduction in any medium, provided the original work is properly cited.

Luminal B breast cancers $(\mathrm{BC})$ have a more aggressive behavior associated with a higher rate of tumor relapse and worse prognosis compared to luminal A tumors. In this study, we evaluated the involvement of specific epithelial-to-mesenchymal transition- (EMT-) and immune-related pathways in the dissemination of luminal B BC cells. The expression of 42 EMT- and immune-related genes was evaluated in matched sentinel lymph nodes (SLNs) analyzed by the one-step nucleic acid amplification assay (OSNA) and primary tumors of 40 luminal B BC patients by gene array and immunohistochemistry. The results were validated in an independent group of 150 luminal B tumors by immunohistochemistry and immunofluorescence and using gene expression data from 315 luminal B BC patients included in the Metabric dataset. We found that the expression of CXCR4 $(p=3.28 E-02)$ and CD163 $(p=6.92 E-03)$ was significantly upregulated in SLNs of recurrent luminal B BC patients. Luminal B primary tumors overexpressing CXCR4 were characterized by an increased expression of vimentin and a high content of CD163-positive macrophages. Bioinformatics analysis confirmed the correlation of CXCR4 with CXCL12, VIM, and CD163 expression and LN involvement. Our results suggest that the upregulation of the CXCR4/CXCL12 pathway and the presence of protumor macrophages in the primary tumor and SLNs sustain the aggressiveness of an important subgroup of luminal B BC.

\section{Introduction}

Breast cancer (BC) is a heterogeneous disease, which encompasses distinct subtypes that differ in molecular features, clinical behavior, and response to treatment [1-3]. Gene expression-based classification identified four major $\mathrm{BC}$ molecular subtypes defined as luminal $\mathrm{A}$ and $\mathrm{B}$, human epidermal growth factor receptor 2- (HER2-) enriched, and triple negative $(\mathrm{TN}) /$ basal-like tumors [1-3]. Luminal B tumors, which account for approximately $20 \%$ of all BC, show a lower expression of estrogen receptor (ER), lower or no expression of progesterone receptor (PgR), and higher proliferation compared to luminal A cancers and can be HER2 positive $[4,5]$. In particular, luminal $B$ tumors have 
a more aggressive behavior compared to luminal A cancers, showing a pattern of tumor recurrence and prognosis similar to those of HER2-enriched and TN/basal-like cancers [6].

It is well established that cancer cells, moving from primary breast tumor, can reach distant organs and metastasize through both blood and lymphatic vessels [7]. The sentinel lymph node (SLN), being by definition the first lymph node reached by $\mathrm{BC}$ cells spreading from the primary site, exerts a pivotal role in disease progression [8]. Tumor dissemination through SLNs, which can be rapidly detected through the one-step nucleic acid amplification assay (OSNA), is often driven by the epithelial-to-mesenchymal transition (EMT) process that allows epithelial cells to detach from the surrounding tissue and acquire a mesenchymal phenotype, gaining migratory and invasive abilities $[9,10]$.

In the last few years, a growing interest has been given to the relationship between tumor cells and the immune system $[11,12]$. Importantly, EMT is emerging as a crucial mechanism regulating the dynamic interactions in the tumor microenvironment and supporting tumor immune escape $[13,14]$. Indeed, cancer cells with mesenchymal features are able to shape the phenotype and the activity of tumorassociated immune cells, which in turn can regulate EMT in cancer cells through the release of multiple soluble mediators $[13,14]$. In particular, inflammatory cells and tumor-associated macrophages (TAMs) have been shown to be able of inducing EMT, sustaining tumor progression in $\mathrm{BC}[13,15]$. Thus, the identification of the mechanisms underlying the acquisition of metastasis-enabling features and the generation of a permissive microenvironment for tumor growth and invasion can help identify luminal B BC patients at high risk of relapse and may represent the rationale for the development of novel therapeutic strategies.

In this study, we evaluated the role of EMT- and immune-related pathways in sustaining the dissemination to SLNs and in driving local and distant relapse in luminal B BC patients.

\section{Methods}

2.1. One-Step Nucleic Acid Amplification (OSNA). The OSNA assay was performed as previously reported, using the OSNA BC System (Sysmex, Kobe, Japan)[16]. Briefly, after removing extranodal and fatty tissues, the SLNs $(\leq 600 \mathrm{mg})$ were homogenized in $4 \mathrm{~mL}$ of Lynorhag lysis buffer (Sysmex) for $90 \mathrm{sec}$ on ice using a Physicotron Warring blender with an NS-4 shaft (MicroTec Nichion) and then centrifuged at $10,000 \times \mathrm{g}$ for $1 \mathrm{~min}$ at room temperature. SLNs exceeding the specified maximum weight of $600 \mathrm{mg}$ were cut into two or more pieces and processed separately. The lysate $(2 \mu \mathrm{L})$ was subjected to the automated reverse transcription loopmediated isothermal amplification (RT-LAMP) of cytokeratin 19 (CK19) mRNA using an RD-100i analyzer (Sysmex). The remaining sample was stored at $-80^{\circ} \mathrm{C}$. CK19 mRNA copy number was determined based on a standard curve generated using a known quantity of human CK19 mRNA, and samples were classified based on the CK19 mRNA copy number $/ \mu \mathrm{L}$. Accordingly, we defined macrometastasis $(++)$ as $>5 \times 10^{3}$ copies $/ \mu \mathrm{L}$ of CK19 mRNA, micrometastasis
$(+)$ as $2.5 \times 10^{2}$ to $5 \times 10^{3}$ copies $/ \mu \mathrm{L}$, and nonmetastasis (-) as $<2.5 \times 10^{2}$ copies $/ \mu \mathrm{L}$. All surgical resection specimens were also analyzed for CK19 expression using immunohistochemistry (RCK108 antibody, Dako).

2.2. Clinical and Pathological Data of Luminal B HER2Negative Breast Cancer. We used a discovery cohort of 40 patients with invasive ductal luminal B (HER2-negative) $\mathrm{BC}$ and OSNA ++ SLNs, who underwent surgery at the Humanitas Clinical and Research Center between 2011 and 2014. The validation dataset included 150 patients with invasive ductal luminal B (HER2-negative) BC and OSNA ++ SLNs, who underwent surgery at the Humanitas Clinical and Research Center between 2006 and 2010. Samples in the two cohorts were homogeneous for all clinicopathological features and treatments. Matched formalinfixed and paraffin-embedded (FFPE) tissues were available for all the selected cases. Patients were classified as relapsing based on the first evidence of invasive relapse at any site. Clinical and pathological data of these cohorts are reported in Supplementary Table 1 and Supplementary Table 2.

\subsection{Sample Processing and Quantitative Reverse Transcription} PCR ( $q R T-P C R)$. The OSNA lysates were incubated at $37^{\circ} \mathrm{C}$ in a water bath until completely thawed and centrifuged at $12,000 \times \mathrm{g}$ for $5 \mathrm{~min}$ at room temperature. The supernatant was discarded, and lysis reagent (Qiazol, Qiagen) was added. RNA was then extracted with the RNeasy Plus Universal Tissue Kit (Qiagen) following the manufacturer's instructions.

RNA retrotranscription was performed with the iScript Advanced cDNA Synthesis Kit (Bio-Rad). Briefly, the reverse transcription master mix was prepared as indicated, and $5 \mu \mathrm{L}$ of total RNA input were added to $15 \mu \mathrm{L}$ of the prepared master mix for each reverse transcription reaction. All the reactions were assembled on ice. The complete reaction mix was then incubated in a thermal cycler according to the manufacturer's protocol.

Based on a comprehensive literature review and pathway enrichment analysis, we identified a set of 42 genes associated with immune and EMT functions (Supplementary Table 3). Expression profiling by qRT-PCR was performed using a custom gene panel (Bio-Rad) using a ViiA 7 Real-Time PCR system (Applied Biosystems). Data were then normalized, and we considered as positive cut-off a $\Delta \mathrm{Ct}>4$.

2.4. Immunohistochemistry and Immunofluorescence. FFPE tissues were cut into $2 \mu \mathrm{m}$ sections and subjected to antigen unmasking at $98^{\circ} \mathrm{C}$ in a water bath for $25 \mathrm{~min}$. Slides were cooled for $30 \mathrm{~min}$ before the treatment with Peroxidase Blocking Reagent (Dako) and Background Sniper (Biocare). Immunohistochemical reactions were performed using CONFIRM anti-Ki67 (clone 30-9; Ventana Medical Systems), CONFIRM anti-vimentin (clone V9; Ventana Medical Systems), anti-CD163 (clone 10D6, 1:1000; Novocastra), anti-CXCR4 (clone UMB2, 1:200; Abcam), and antiCXCL12 (clone 79018, 1:500; R\&D Systems) primary antibodies. DAB chromogen kit (Biocare Medical) was used for immunodetection. All analyses were performed centrally at the Humanitas Clinical and Research Center 
by two pathologists, who were blinded for patient characteristics and outcome. The staining was evaluated by intensity $(0=$ no staining, $1=$ weak, $2=$ moderate, and $3=$ strong) and the percentage of stained cells $(0=0 \%$, $1=1-10 \%, 2=11-50 \%, 3=51-80 \%$, and $4>80 \%)$. The immunoreactive score (IRS) was obtained by multiplying the intensity with the proportion of positive cells, and samples were classified as negative $(\operatorname{IRS}=0-5)$ or positive $(\mathrm{IRS}=6-12)$ [17-19]. CD163 staining was scored using a four-tiered system ranging from 0 (absent) to 3 (dense), as previously described [15].

For immunofluorescence, FFPE sections $(3 \mu \mathrm{m})$ were subjected to UV radiation for $48 \mathrm{~h}$ at $16^{\circ} \mathrm{C}$. Deparaffinization and antigen unmasking were performed in sodium citrate buffer pH6.0 (Bio-Optica) at $98^{\circ} \mathrm{C}$ in a water bath for $25 \mathrm{~min}$. Slides were cooled in deionized water and blocked with PBS containing $2 \%$ bovine serum albumin and $2 \%$ goat serum for $30 \mathrm{~min}$ at room temperature. Sections were then incubated with anti-CXCR4 antibody (clone UMB2, $1: 200$; Abcam) for $1 \mathrm{~h}$ at room temperature and then with antiCD163 antibody (clone 10D6, 1:1000; Novocastra) for $1 \mathrm{~h}$ at room temperature. Goat anti-rabbit Alexa Fluor 488 and goat anti-mouse Alexa Fluor 594 (Invitrogen) were used as secondary antibodies. Slides were counterstained with DAPI and mounted with ProLong Gold (Invitrogen). Images were captured using an Olympus Fluoview FV1000 laser scanning confocal microscope (Olympus).

2.5. In Silico Data Analysis. Gene expression data from the Metabric dataset were processed as previously described [20, 21]. Overall, we analyzed 315 patients with luminal B (HER2-negative) BC, as defined by the PAM50 classifier and based on the expression of the ERBB2 gene. Patients' characteristics are reported in Supplementary Table 4.

2.6. Statistical Analysis. The analysis of differential gene expression between patients' subgroups was performed by unpaired two-tailed $t$-test. Spearman's rank correlation test was used to evaluate the correlation between variables. Clinicopathological associations were investigated using Fisher's exact test (FET) and Mann-Whitney $U$ test for categorical and continuous data, respectively. For categorical analysis, the median expression of CXCR4 was used as cut-off. $p$ values were adjusted for multiple testing by Benjamini-Hochberg correction, and the level of statistical significance was set at $p<0.05$.

\section{Results}

3.1. Identification of Genes Differentially Expressed between Relapsing and Nonrelapsing Luminal B BC Patients. We first investigated the potential involvement of EMT and immune signaling in the metastatic dissemination of luminal B BC. We analyzed the expression of 42 genes associated with these pathways in 40 OSNA ++ SLNs of luminal B (HER2-negative) $\mathrm{BC}$ patients, which included 20 relapsing subjects and 20 tumor recurrence-free patients (Supplementary Table 1).

We identified 12 genes that were differentially expressed between recurrent and nonrecurrent luminal B BC patients
TABLE 1: Genes differentially expressed in the OSNA ++ sentinel lymph nodes of relapsing and nonrelapsing luminal B BC patients.

\begin{tabular}{|c|c|c|c|}
\hline Gene & Fold change & $p$ value & Adjusted $p$ value* \\
\hline TGFB2 & 5.2 & $7.81 E-05$ & $3.28 E-03$ \\
\hline CD163 & 5.5 & $3.30 E-04$ & $6.92 E-03$ \\
\hline COL1A2 & 8.3 & $3.40 E-03$ & $3.28 E-02$ \\
\hline CXCR4 & 3.4 & $4.17 E-03$ & $3.28 E-02$ \\
\hline ITGAV & 2.9 & $4.25 E-03$ & $3.28 E-02$ \\
\hline TWIST1 & 5.2 & $4.69 E-03$ & $3.28 E-02$ \\
\hline CALD1 & 4.1 & $6.44 E-03$ & $3.59 E-02$ \\
\hline SNAI2 & 3.7 & $6.84 E-03$ & $3.59 E-02$ \\
\hline MKI67 & 1.9 & $1.26 E-02$ & $5.24 E-02$ \\
\hline$B M P 1$ & 0.4 & $1.27 E-02$ & $5.24 E-02$ \\
\hline$L A G 3$ & 3.2 & $1.40 E-02$ & $5.24 E-02$ \\
\hline $\mathrm{CDH} 2$ & 2.5 & $1.50 E-02$ & $5.24 E-02$ \\
\hline IL2 & 3.4 & $1.78 E-02$ & $5.68 E-02$ \\
\hline STEAP1 & 5.8 & $1.89 E-02$ & $5.68 E-02$ \\
\hline ZEB1 & 2.8 & $2.77 E-02$ & $7.76 E-02$ \\
\hline FOXC2 & 7.6 & $3.32 E-02$ & $8.73 E-02$ \\
\hline$P G R$ & 4.3 & $3.71 E-02$ & $9.16 E-02$ \\
\hline $\mathrm{CDH} 1$ & 2.2 & $5.20 E-02$ & $1.21 E-01$ \\
\hline VCAN & 2.3 & $5.53 E-02$ & $1.22 E-01$ \\
\hline WNT5A & 2.0 & $7.42 E-02$ & $1.49 E-01$ \\
\hline HPRT1 & 1.8 & $7.46 E-02$ & $1.49 E-01$ \\
\hline CD28 & 2.2 & $9.66 E-02$ & $1.84 E-01$ \\
\hline TMEFF1 & 2.7 & $1.15 E-01$ & $2.09 E-01$ \\
\hline GSC & 2.7 & $1.33 E-01$ & $2.33 E-01$ \\
\hline SNAI1 & 2.6 & $1.53 E-01$ & $2.57 E-01$ \\
\hline OSM & 2.7 & $1.67 E-01$ & $2.69 E-01$ \\
\hline IL10 & 2.3 & $1.79 E-01$ & $2.78 E-01$ \\
\hline$F G F 1$ & 2.2 & $1.90 E-01$ & $2.80 E-01$ \\
\hline CSF1 & 1.9 & $1.93 E-01$ & $2.80 E-01$ \\
\hline AHNAK & 0.8 & $2.30 E-01$ & $3.21 E-01$ \\
\hline SOX10 & 2.5 & $2.54 E-01$ & $3.43 E-01$ \\
\hline TIMP1 & 1.4 & $3.11 E-01$ & $4.08 E-01$ \\
\hline ESR1 & 1.5 & $4.31 E-01$ & $5.48 E-01$ \\
\hline VIM & 1.2 & $4.58 E-01$ & $5.66 E-01$ \\
\hline$I G F 1 R$ & 1.3 & $5.06 E-01$ & $6.07 E-01$ \\
\hline TGFB1 & 0.8 & $5.37 E-01$ & $6.27 E-01$ \\
\hline SPARC & 0.9 & $5.99 E-01$ & $6.80 E-01$ \\
\hline IGFBP4 & 1.1 & $6.57 E-01$ & $7.26 E-01$ \\
\hline$E R B B 2$ & 1.2 & $7.07 E-01$ & $7.61 E-01$ \\
\hline$C D 4$ & 1.1 & $8.42 E-01$ & $8.69 E-01$ \\
\hline$A X L$ & 1.1 & $8.48 E-01$ & $8.69 E-01$ \\
\hline$M R C 1$ & 1.0 & $9.00 E-01$ & $9.00 E-01$ \\
\hline
\end{tabular}

${ }^{*}$ Benjamini-Hochberg-adjusted $p$ values.

(Table 1). However, only eight genes (CALD1, CD163, COL1A2, CXCR4, ITGAV, SNAI2, TGFB2, and TWIST1), whose expression was upregulated in SLNs of patients who experienced tumor relapse, remained significant after 
Relapse versus relapse-free

CXCR4

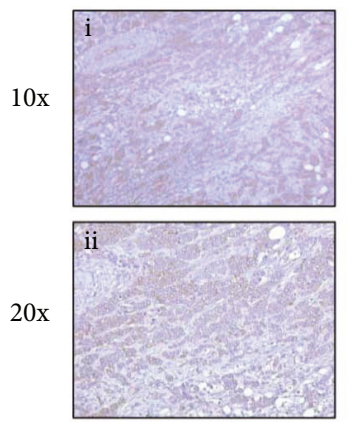

(a)
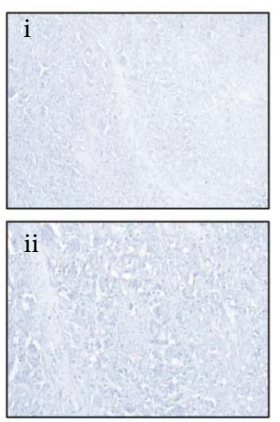

Relapse versus relapse-free

CXCL12

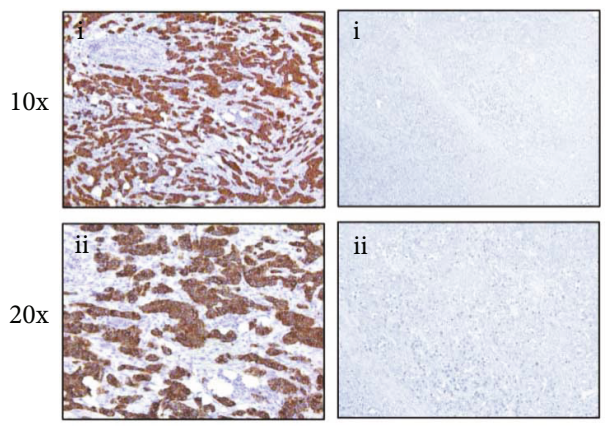

(b)

Figure 1: CXCR4 and CXCL12 expression in luminal B BC samples. (a) Immunohistochemical analysis showing a higher expression of CXCR4 protein in the primary tumors of relapsing compared to relapse-free luminal B BC patients. (b) Immunohistochemical analysis of CXCL12 protein levels in the primary tumors of relapsing and relapse-free luminal B BC patients. (i) 10x magnification and (ii) 20x magnification.

adjustment for multiple testing (Table 1), indicating that both EMT and immune response have a crucial role in the spread of luminal B BC cells to SLNs. In particular, the chemokine receptor CXCR4, which mediates both the EMT process and the polarization toward an immunosuppressive microenvironment, has been directly involved in the progression of ER-positive BC [22-24]. Furthermore, the high expression of CD163 in SLNs from relapsing patients may suggest an active role of $\mathrm{M} 2$ protumor macrophages in supporting the aggressiveness of luminal B BC cells.

3.2. CXCR4/CXCL12 Axis Has a Key Role in Determining the Metastatic Destination of Luminal B BC. To evaluate the potential involvement of CXCR4- and CD163-positive macrophages in the progression of luminal $\mathrm{B} B C$, we analyzed the matched primary tumors of luminal B BC patients in the discovery cohort. We found that CXCR4 protein was overexpressed in relapsing luminal B BC patients compared with nonrelapsing cases (FET $p=0.010$; Figure 1(a)). Furthermore, we showed that primary tumors with significant high levels of the CXCR4 receptor also overexpressed its ligand CXCL12 (Figure 1(b)). The association between CXCR4 protein levels and tumor relapse (FET $p=0.031$ ) was confirmed in luminal B BC patients of the validation cohort (FET $p=0.031$; Figure 2). Importantly, we demonstrated that luminal B primary tumors overexpressing CXCR4 were characterized by an increased expression of the mesenchymal marker vimentin and by a high content of CD163-positive TAMs (FET $p=0.033$; Figure 2). Conversely, we were not able to detect any association between the expression of CXCR4 and Ki67 in luminal B BC (Figure 2).

To confirm the relevance of TAMs in favoring the aggressiveness of BC cells, we performed double immunofluorescence for CXCR4 and CD163 on tumor tissues of luminal B $\mathrm{BC}$ patients from the validation cohort. Accordingly, we demonstrated that CXCR4-positive BC cells and CD163positive TAMs were localized in the same tumor regions in
$75 \%$ of relapsing cases, while no association was observed in nonrelapsing luminal B cancers (Figure 3).

These findings were further validated by analyzing the gene expression profiles of 315 luminal B (HER2-negative) $\mathrm{BC}$ patients enclosed in the Metabric dataset. In silico analysis confirmed that the expression of CXCR4 in the primary tumor significantly correlated not only with the expression of its ligand CXCL12 (Spearman's coefficient, rs $=0.4920$ ) but also with the expression of VIM ( $\mathrm{rs}=0.4779$ ) and CD163 (rs = 0.4853) (Figure 4(a)). Similarly, the expression of CXCL12 was strongly associated with the expression of VIM (rs=0.7133) and CD163 (rs =0.4454) (Figure 4(b)). Conversely, we found a mild inverse correlation of CXCR4 and CXCL12 with MKI67 (rs $=-0.1262$; rs $=-0.2076$, resp.), thus confirming the lack of association between the CXCR4/CXCL12 axis and proliferation in luminal B BC (Figures 4(a) and 4(b)). Furthermore, we demonstrated that the overexpression of CXCR4 in luminal B (HER2-negative) BC was significantly associated with LN positivity according to both Mann-Whitney $(p=0.003)$ and FET tests $(p=0.001)$.

On overall, these results suggest that a permissive tumor microenvironment in the primary tumor and SLNs, characterized by EMT features such as the activation of CXCR4/ CXCL12 axis and the presence of protumor M2 TAMs, can sustain the aggressiveness of cancer cells and support their metastatic dissemination in luminal B BC.

\section{Discussion}

Luminal cancers, which enclose around two-thirds of all BC, are generally considered less aggressive and associated with better prognosis compared with nonluminal tumors. However, luminal B cancers are recognized as having a metastatic dissemination time pattern and an outcome similar to those of HER2-positive and TNBC, with an increased risk of tumor relapse in the first five years after diagnosis [6]. SLN biopsy has been demonstrated to be an accurate predictor of axillary LN status, which is considered a consistent 


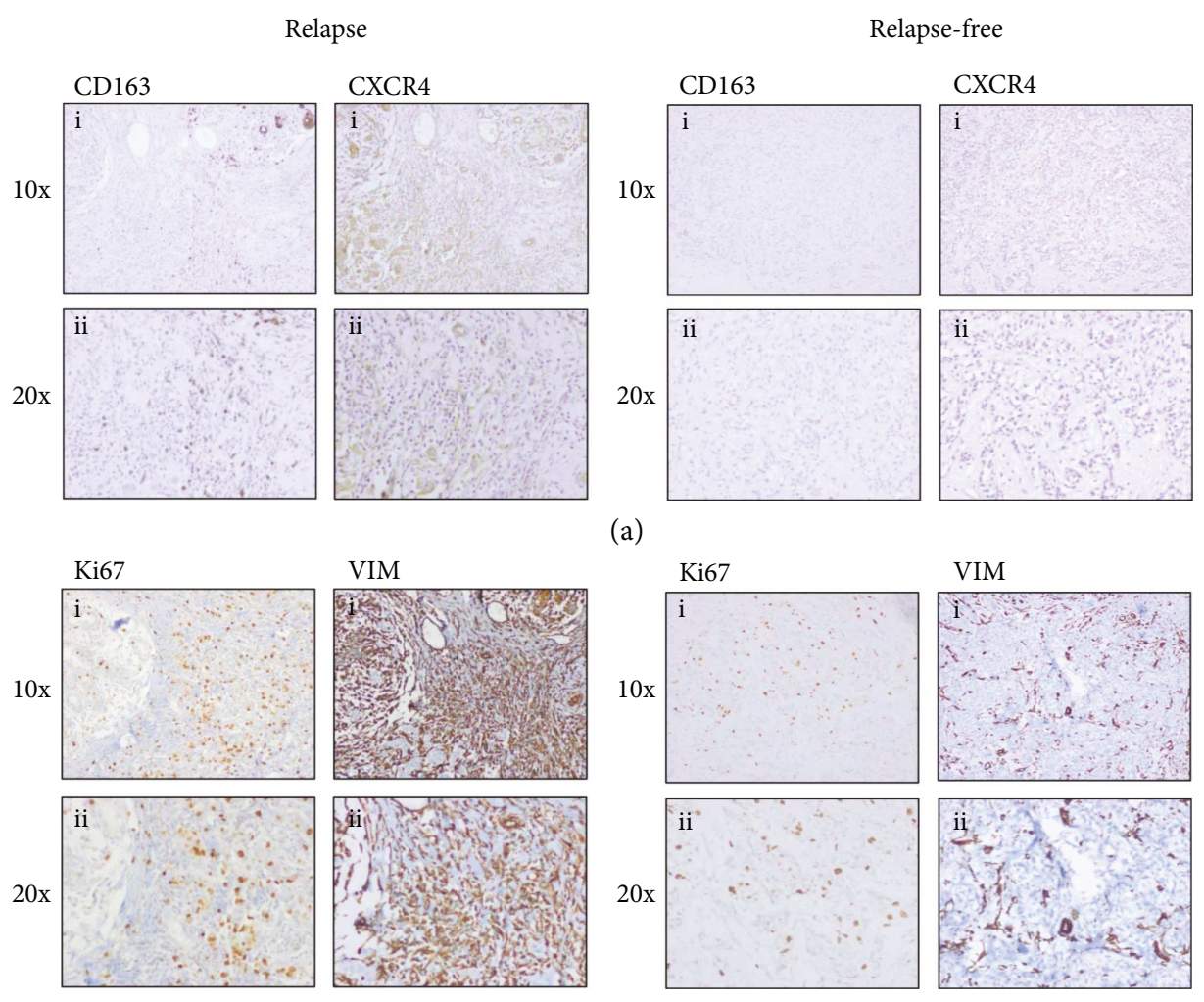

(b)

Figure 2: Immunohistochemical staining for CXCR4, vimentin, and CD163-positive macrophages in luminal B BC samples. (a) Immunohistochemical analysis of CXCR4 protein levels and the presence of CD163-positive macrophages in the primary tumors of relapsing and relapse-free luminal B BC patients. (b) Evaluation of Ki67 and vimentin protein expression in the primary tumors of relapsing and relapse-free luminal B BC patients. (i) 10x magnification and (ii) 20x magnification.
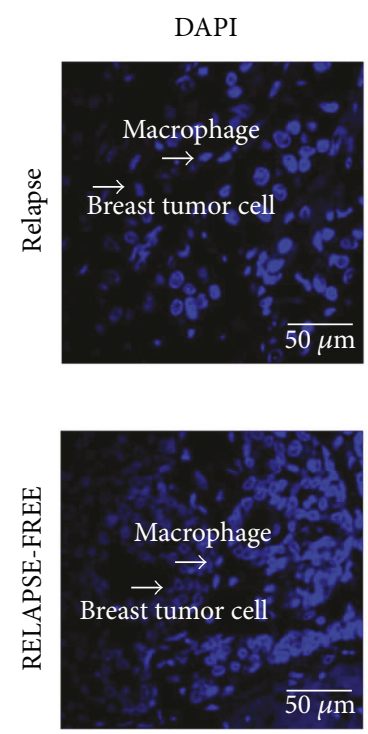
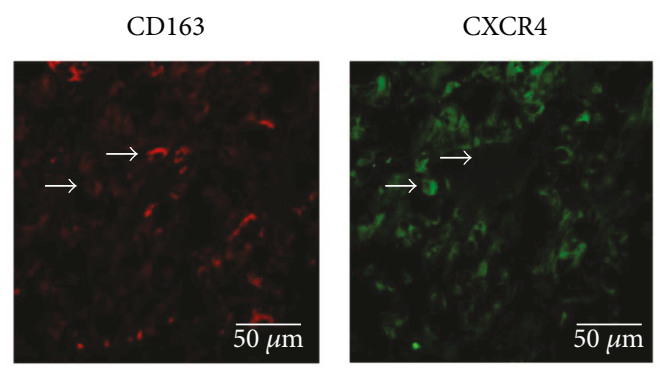

(a)
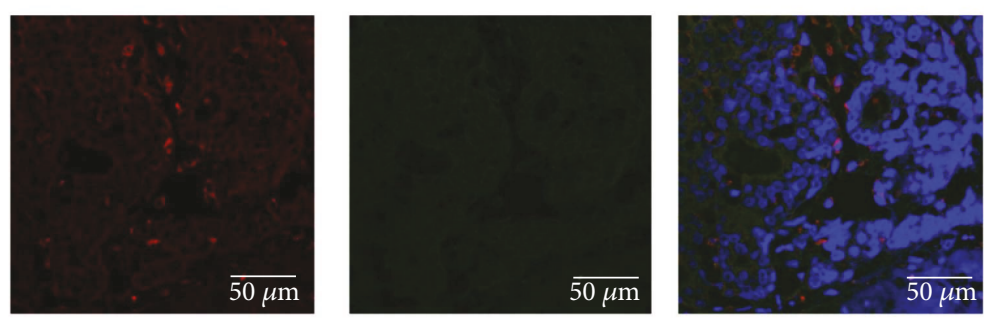

(b)

Figure 3: Double immunofluorescent staining for CXCR4 and CD163 in luminal B BC samples. Representative images of double immunofluorescent staining and confocal microscopy on primary tumors of relapsing (a) and nonrelapsing (b) luminal B BC patients showing that CXCR4-expressing cancer cells (green) and CD163-positive TAMs (red) are localized in the same tumor regions of relapsing luminal B BC. Scale bars represent $50 \mu \mathrm{m}$. 

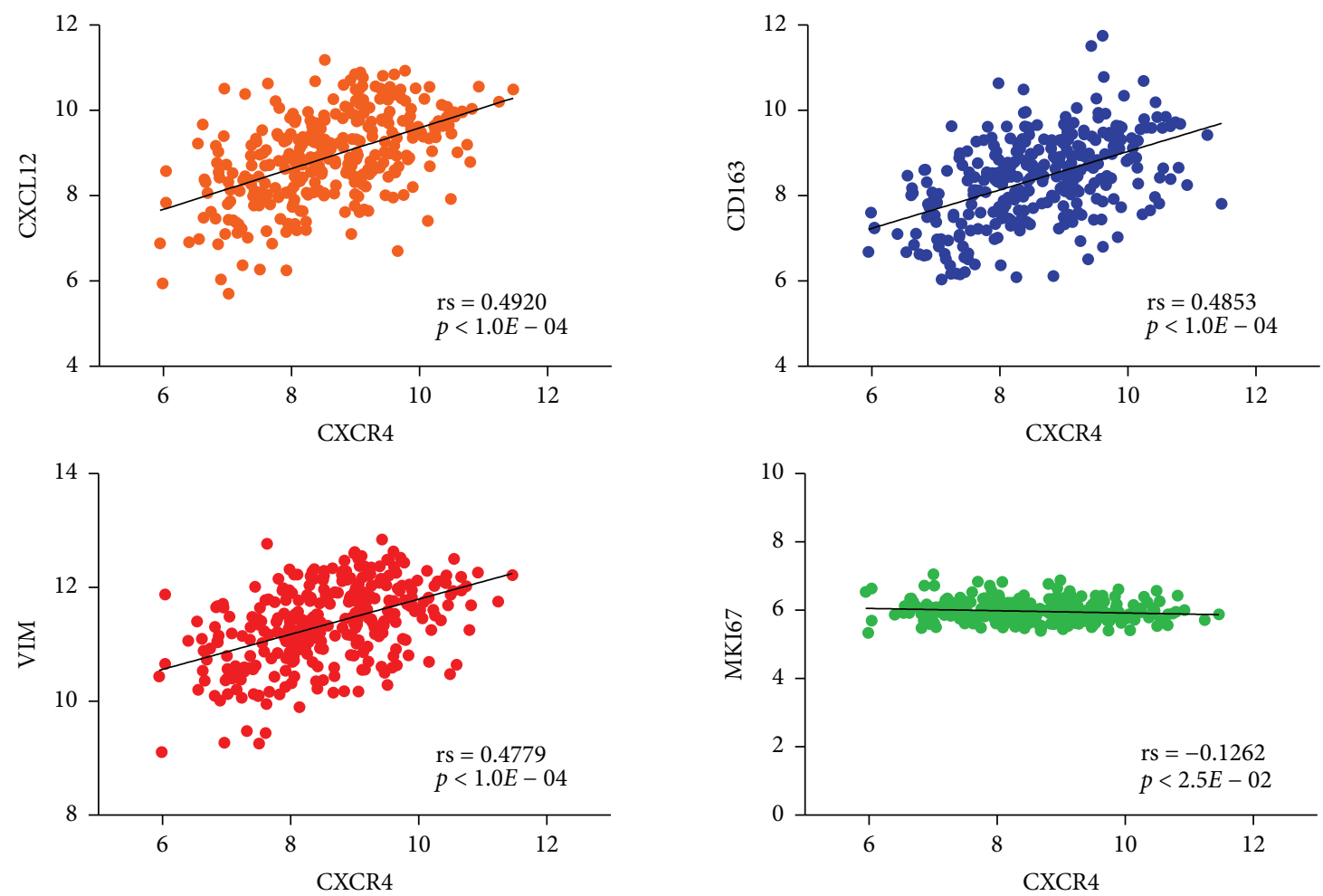

(a)
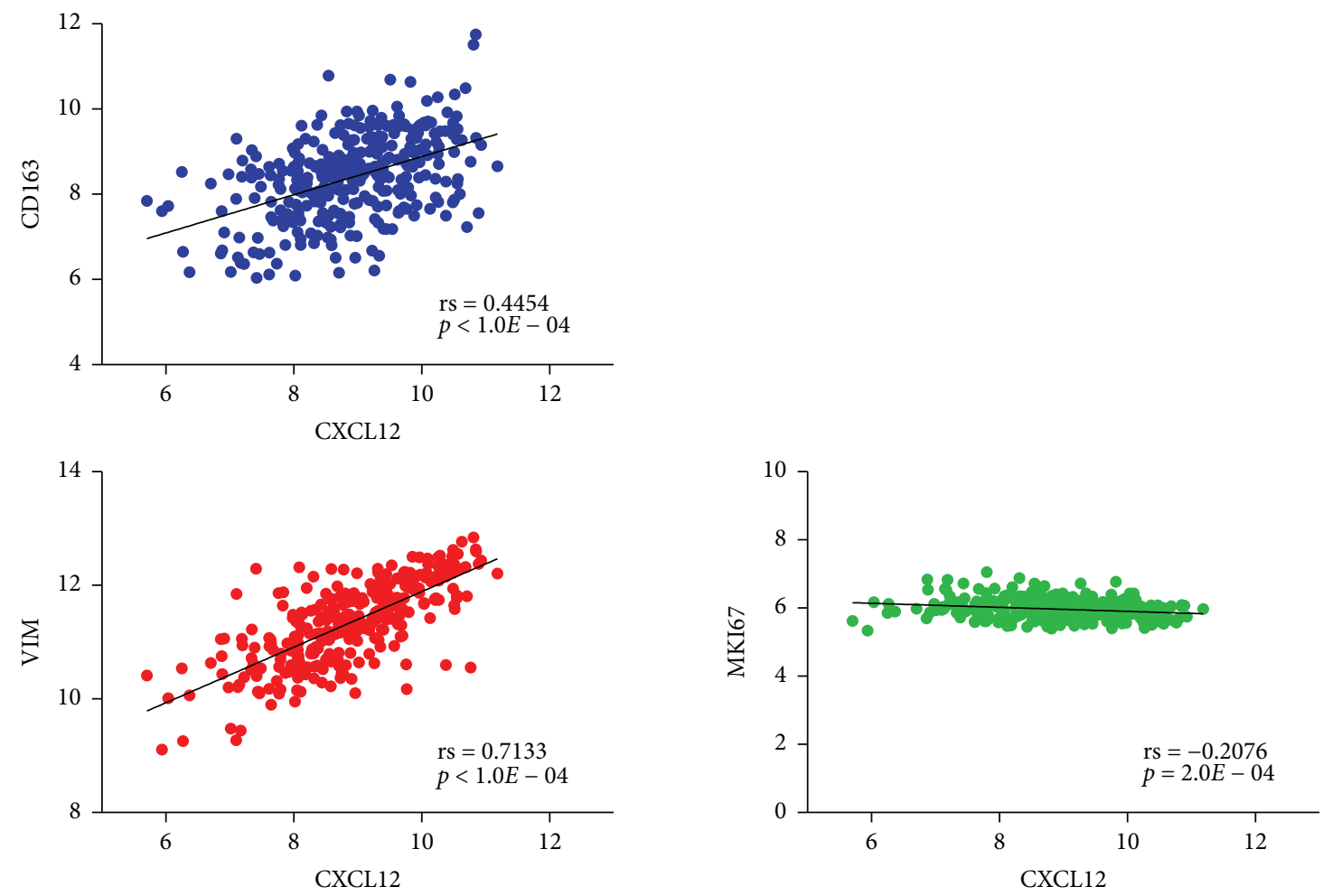

(b)

FIgURe 4: In silico analysis of gene expression data of luminal B BC patients from the Metabric dataset. (a) Correlation between CXCR4, CXCL12, VIM, CD163, and MKI67 in 315 luminal B (HER2-negative) primary BC. (b) Correlation between CXCL12, VIM, CD163, and MKI67 in 315 luminal B (HER2-negative) primary BC. Spearman's coefficients (rs) and $p$ values are shown. 
prognostic factor in $\mathrm{BC}$ including luminal $\mathrm{B}$ tumors and it is currently used in treatment decision-making [25-28].

Migration of primary tumor cells in the local lymphatic system facilitates the dissemination to the SLN and subsequently to distant organs. EMT and immunosuppression are increasingly recognized as potent inducers of tumor progression and invasion, although the mechanisms mediating the tumor spread to the regional draining $\mathrm{LN}$ are still not fully elucidated [9-15]. In this study, we demonstrated that the CXCR4/CXCL12 pathway was upregulated in the primary tumor and in the matched macrometastatic SLNs of luminal B BC patients who had tumor recurrence, and it was associated with characteristics of tumor aggressiveness and invasiveness such as LN involvement. Increased levels of CXCR4/CXCL12 have been found in many types of cancer, including BC, and have been associated with metastasis and poor prognosis [22, 29-32]. Accordingly, a strong expression of CXCR4 has been demonstrated in primary breast tumors, axillary LN metastases, and distant metastases of the lung and liver [30]. Interestingly, we found that the expression of CXCR4 correlated with the levels of its ligand CXCL12 in the primary tumors of relapsing luminal B BC patients. In agreement with previous findings showing that CXCL12 is preferentially expressed in the most common metastatic sites of BC (i.e., the LNs, lung, liver, and bone marrow) and that it induces the recruiting of CXCR4-positive cancer cells to CXCL12-expressing sites, our results suggest that the chemokine CXCL12 and its cognate receptor CXCR4 exert a key role in determining the metastatic potential of breast tumor cells [30, 33].

In ER-positive $\mathrm{BC}$, the activation of CXCR4 signaling has been demonstrated to drive $\mathrm{BC}$ cells to an invasive and endocrine therapy-resistant phenotype through the activation of extracellular signal-regulated kinases (ERK) 1/2, p38 mitogen-activated protein kinase (MAPK), and NF- $\kappa \mathrm{B}$ pathways and through the enhancement of ER-mediated gene expression [23, 24, 34]. Furthermore, CXCR4/CXCL12 axis has been shown to be capable of inducing EMT in ERpositive BC through the regulation of key EMT markers such as E-cadherin and $\mathrm{N}$-cadherin [23, 24]. Accordingly, we demonstrated that the CXCR4 pathway correlated with the expression of the mesenchymal marker vimentin in luminal $\mathrm{B}$ primary tumors and that the overexpression of CXCR4 in metastatic SLNs was concurrent with that of other EMTrelated genes, such as SNAI2, TGFB2, and TWIST1. Thus, our results provide an important evidence for the association of CXCR4/CXCL12 axis with the progression of tumor cells toward a mesenchymal phenotype, suggesting a potential mechanism that drives the metastatic spread of luminal B BC cells from primary tumors and SLNs to distant sites.

Growing evidence indicates that the metastatic ability of a cancer relies on intrinsic properties of tumor cells such as EMT and on signals derived from the tumor microenvironment [35]. Interestingly, numerous chemokines and associated receptors, including CXCL12 and CXCR4, have been shown to exert a key role in mediating the communication between cancer cells and nonmalignant stromal cells, ultimately favoring the establishment of a permissive microenvironment for tumor development and progression $[22,30,36]$. Accordingly, we found that CD163 was overexpressed in metastatic SLNs of relapsing luminal B BC patients. Furthermore, we demonstrated that aggressive luminal B primary tumors characterized by the high expression of CXCR4 and CXCL12 showed an increased content of CD163-positive macrophages. Additionally, we revealed that protumor TAMs and CXCR4-positive cancer cells were frequently localized in the same areas of the primary tumor. Of note, TAMs, which essentially resemble alternatively activated M2-like macrophages, have been shown to affect multiple aspects of cancer progression, including EMT and immune suppression, and to modulate the response to anticancer therapies [15, 37, 38]. Moreover, mononuclear phagocytes are attracted to tumor and metastatic sites by the presence of CXCL12, which is able to shape monocyte polarization toward a protumor M2-like phenotype [36, 39-41].

\section{Conclusions}

In conclusion, although further studies are required to validate the molecular mechanisms and the association between the expression of these EMT- and immune-related markers and $\mathrm{BC}$ progression, our findings suggest that the upregulation of the CXCR4/CXCL12 axis and the presence of protumor macrophages in the primary tumor and SLNs sustain the aggressiveness of luminal B BC cells, favoring the generation of a permissive tumor microenvironment and leading to metastatic spread.

\section{Conflicts of Interest}

The authors declare that they have no conflict of interest.

\section{Acknowledgments}

Giulia Bottai is supported by Fondazione Italiana Ricerca sul Cancro (FIRC fellowship 18328).

\section{Supplementary Materials}

Supplementary Table 1: clinical and pathological features of patients enclosed in the discovery cohort $(n=40)$. Supplementary Table 2: clinical and pathological features of patients enclosed in the validation cohort $(n=150)$. Supplementary Table 3: detailed list of the investigated genes. Supplementary Table 4: clinical and pathological features of patients enclosed in the Metabric cohort. (Supplementary Materials)

\section{References}

[1] Cancer Genome Atlas Network, "Comprehensive molecular portraits of human breast tumours," Nature, vol. 490, no. 7418, pp. 61-70, 2012.

[2] C. M. Perou, T. Sorlie, M. B. Eisen et al., "Molecular portraits of human breast tumours," Nature, vol. 406, no. 6797, pp. 747-752, 2000.

[3] C. Sotiriou and L. Pusztai, "Gene-expression signatures in breast cancer," The New England Journal of Medicine, vol. 360, no. 8, pp. 790-800, 2009. 
[4] C. Fan, D. S. Oh, L. Wessels et al., "Concordance among geneexpression-based predictors for breast cancer," The New England Journal of Medicine, vol. 355, no. 6, pp. 560-569, 2006.

[5] K. David Voduc, M. C. U. Cheang, S. Tyldesley, K. Gelmon, T. O. Nielsen, and H. Kennecke, "Breast cancer subtypes and the risk of local and regional relapse," Journal of Clinical Oncology, vol. 28, no. 10, pp. 1684-1691, 2010.

[6] F. Ades, D. Zardavas, I. Bozovic-Spasojevic et al., "Luminal B breast cancer: molecular characterization, clinical management, and future perspectives," Journal of Clinical Oncology, vol. 32, no. 25, pp. 2794-2803, 2014.

[7] S. A. Stacker, S. P. Williams, T. Karnezis, R. Shayan, S. B. Fox, and M. G. Achen, "Lymphangiogenesis and lymphatic vessel remodelling in cancer," Nature Reviews Cancer, vol. 14, no. 3, pp. 159-172, 2014.

[8] S. Ran, L. Volk, K. Hall, and M. J. Flister, "Lymphangiogenesis and lymphatic metastasis in breast cancer," Pathophysiology, vol. 17, no. 4, pp. 229-251, 2010.

[9] S. Lamouille, J. Xu, and R. Derynck, "Molecular mechanisms of epithelial-mesenchymal transition," Nature Reviews Molecular Cell Biology, vol. 15, no. 3, pp. 178-196, 2014.

[10] K. Polyak and R. A. Weinberg, "Transitions between epithelial and mesenchymal states: acquisition of malignant and stem cell traits," Nature Reviews Cancer, vol. 9, no. 4, pp. 265-273, 2009.

[11] G. P. Dunn, A. T. Bruce, H. Ikeda, L. J. Old, and R. D. Schreiber, "Cancer immunoediting: from immunosurveillance to tumor escape," Nature Immunology, vol. 3, no. 11, pp. 991998, 2002.

[12] G. P. Dunn, L. J. Old, and R. D. Schreiber, "The three Es of cancer immunoediting," Annual Review of Immunology, vol. 22, no. 1, pp. 329-360, 2004.

[13] P. J. Chockley and V. G. Keshamouni, "Immunological consequences of epithelial-mesenchymal transition in tumor progression," The Journal of Immunology, vol. 197, no. 3, pp. 691-698, 2016.

[14] J. M. Reiman, K. L. Knutson, and D. C. Radisky, "Immune promotion of epithelial-mesenchymal transition and generation of breast cancer stem cells," Cancer Research, vol. 70, no. 8, pp. 3005-3008, 2010.

[15] G. Bottai, C. Raschioni, B. Székely et al., "AXL-associated tumor inflammation as a poor prognostic signature in chemotherapy-treated triple-negative breast cancer patients," npj Breast Cancer, vol. 2, no. 1, article 16033, 2016.

[16] S. Klingler, F. Marchal, P. Rauch et al., "Using one-step nucleic acid amplification (OSNA) for intraoperative detection of lymph node metastasis in breast cancer patients avoids second surgery and accelerates initiation of adjuvant therapy," Annals of Oncology, vol. 24, no. 9, pp. 2305-2309, 2013.

[17] Y. Sun, X. Mao, C. Fan et al., "CXCL12-CXCR4 axis promotes the natural selection of breast cancer cell metastasis," Tumour Biology, vol. 35, no. 8, pp. 7765-7773, 2014.

[18] T. Thomaidis, A. Maderer, S. E. Al-Batran et al., "VEGFR-3 and CXCR4 as predictive markers for treatment with fluorouracil, leucovorin plus either oxaliplatin or cisplatin in patients with advanced esophagogastric cancer: a comparative study of the Arbeitsgemeinschaft Internistische Onkologie (AIO)," BMC Cancer, vol. 14, no. 1, p. 476, 2014.

[19] R. A. Werner, A. Weich, T. Higuchi et al., "Imaging of chemokine receptor 4 expression in neuroendocrine tumors - a triple tracer comparative approach," Theranostics, vol. 7, no. 6, pp. 1489-1498, 2017.

[20] C. Curtis, S. P. Shah, S.-F. Chin et al., "The genomic and transcriptomic architecture of 2,000 breast tumours reveals novel subgroups," Nature, vol. 486, pp. 346-352, 2012.

[21] B. Gyorffy, G. Bottai, T. Fleischer et al., "Aberrant DNA methylation impacts gene expression and prognosis in breast cancer subtypes," International Journal of Cancer, vol. 138, no. 1, pp. 87-97, 2016.

[22] F. Guo, Y. Wang, J. Liu, S. C. Mok, F. Xue, and W. Zhang, "CXCL12/CXCR4: a symbiotic bridge linking cancer cells and their stromal neighbors in oncogenic communication networks," Oncogene, vol. 35, no. 7, pp. 816-826, 2016.

[23] L. V. Rhodes, M. R. Bratton, Y. Zhu et al., "Effects of SDF-1CXCR4 signaling on microRNA expression and tumorigenesis in estrogen receptor-alpha (ER- $\alpha$ )-positive breast cancer cells," Experimental Cell Research, vol. 317, no. 18, pp. 2573-2581, 2011.

[24] L. V. Rhodes, S. P. Short, N. F. Neel et al., "Cytokine receptor CXCR4 mediates estrogen-independent tumorigenesis, metastasis, and resistance to endocrine therapy in human breast cancer," Cancer Research, vol. 71, no. 2, pp. 603-613, 2011.

[25] M. Cianfrocca and L. J. Goldstein, "Prognostic and predictive factors in early-stage breast cancer," The Oncologist, vol. 9, no. 6, pp. 606-616, 2004.

[26] G. S. Liao, Y. C. Chou, H. M. Hsu, M. S. Dai, and J. C. Yu, “The prognostic value of lymph node status among breast cancer subtypes," The American Journal of Surgery, vol. 209, no. 4, pp. 717-724, 2015.

[27] E. Senkus, S. Kyriakides, F. Penault-Llorca et al., "Primary breast cancer: ESMO Clinical Practice Guidelines for diagnosis, treatment and follow-up," Annals of Oncology, vol. 24, Supplement 6, pp. vi7-vi23, 2013.

[28] U. Veronesi, G. Paganelli, G. Viale et al., "Sentinel-lymphnode biopsy as a staging procedure in breast cancer: update of a randomised controlled study," The Lancet Oncology, vol. 7, no. 12, pp. 983-990, 2006.

[29] K. E. Luker and G. D. Luker, "Functions of CXCL12 and CXCR4 in breast cancer," Cancer Letters, vol. 238, no. 1, pp. 30-41, 2006.

[30] A. Muller, B. Homey, H. Soto et al., "Involvement of chemokine receptors in breast cancer metastasis," Nature, vol. 410, no. 6824, pp. 50-56, 2001.

[31] T. Sobolik, Y. J. Su, S. Wells, G. D. Ayers, R. S. Cook, and A. Richmond, "CXCR4 drives the metastatic phenotype in breast cancer through induction of CXCR2 and activation of MEK and PI3K pathways," Molecular Biology of the Cell, vol. 25, no. 5, pp. 566-582, 2014.

[32] Z. Zhang, C. Ni, W. Chen et al., "Expression of CXCR4 and breast cancer prognosis: a systematic review and metaanalysis," BMC Cancer, vol. 14, no. 1, p. 49, 2014.

[33] E. R. Pereira, D. Jones, K. Jung, and T. P. Padera, “The lymph node microenvironment and its role in the progression of metastatic cancer," Seminars in Cell \& Developmental Biology, vol. 38, pp. 98-105, 2015.

[34] F. R. Balkwill, "The chemokine system and cancer," The Journal of Pathology, vol. 226, no. 2, pp. 148-157, 2012.

[35] D. Hanahan and R. A. Weinberg, "Hallmarks of cancer: the next generation," Cell, vol. 144, no. 5, pp. 646-674, 2011.

[36] S. V. Kozin, W. S. Kamoun, Y. Huang, M. R. Dawson, R. K. Jain, and D. G. Duda, "Recruitment of myeloid but not 
endothelial precursor cells facilitates tumor regrowth after local irradiation," Cancer Research, vol. 70, no. 14, pp. 5679-5685, 2010.

[37] S. K. Biswas, P. Allavena, and A. Mantovani, "Tumorassociated macrophages: functional diversity, clinical significance, and open questions," Seminars in Immunopathology, vol. 35, no. 5, pp. 585-600, 2013.

[38] A. Mantovani and P. Allavena, "The interaction of anticancer therapies with tumor-associated macrophages," Journal of Experimental Medicine, vol. 212, no. 4, pp. 435-445, 2015.

[39] F. O. Martinez, S. Gordon, M. Locati, and A. Mantovani, "Transcriptional profiling of the human monocyte-tomacrophage differentiation and polarization: new molecules and patterns of gene expression," The Journal of Immunology, vol. 177, no. 10, pp. 7303-7311, 2006.

[40] A. Rigo, M. Gottardi, A. Zamo et al., "Macrophages may promote cancer growth via a GM-CSF/HB-EGF paracrine loop that is enhanced by CXCL12," Molecular Cancer, vol. 9, no. 1, p. 273, 2010.

[41] L. Sanchez-Martin, A. Estecha, R. Samaniego, S. SanchezRamon, M. A. Vega, and P. Sanchez-Mateos, "The chemokine CXCL12 regulates monocyte-macrophage differentiation and RUNX3 expression," Blood, vol. 117, no. 1, pp. 88-97, 2011. 


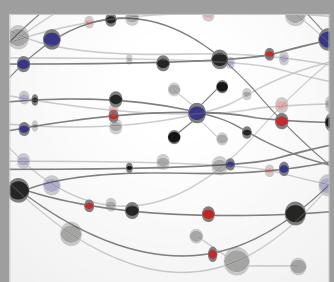

The Scientific World Journal
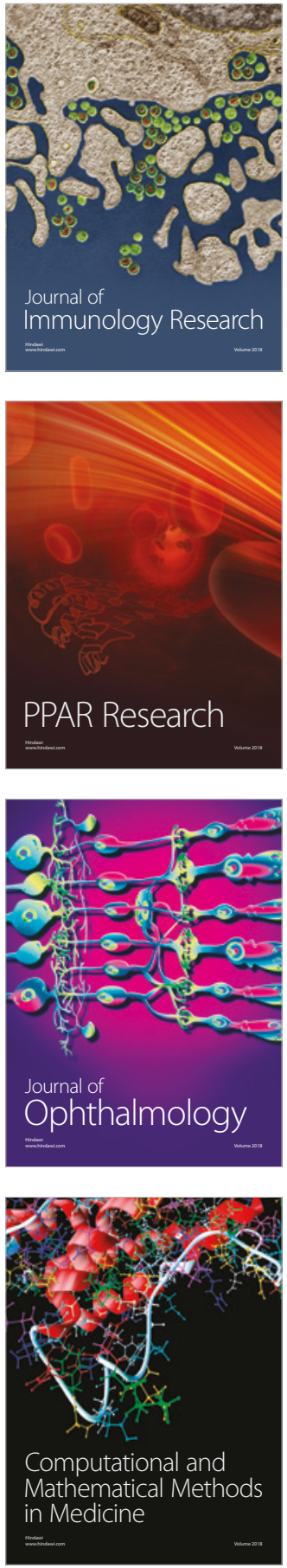

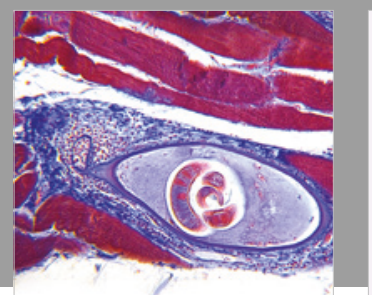

Gastroenterology Research and Practice

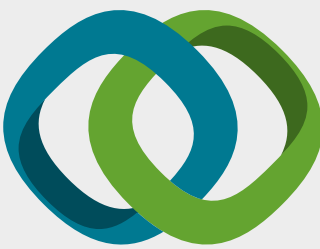

\section{Hindawi}

Submit your manuscripts at

www.hindawi.com
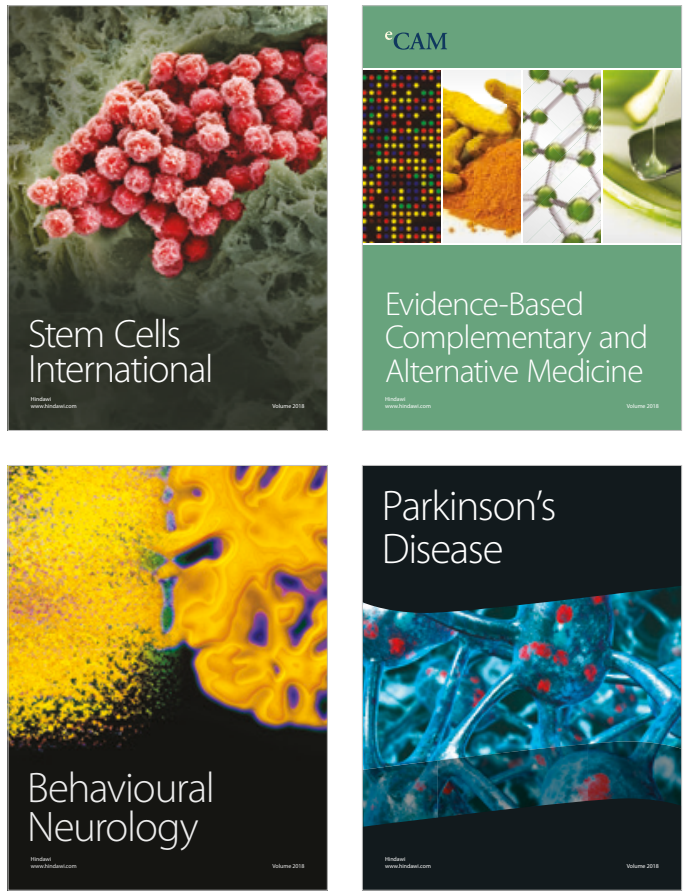

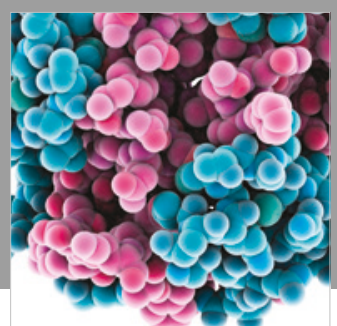

ournal of

Diabetes Research

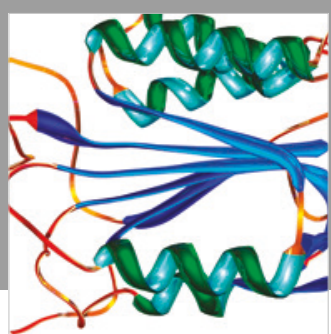

Disease Markers
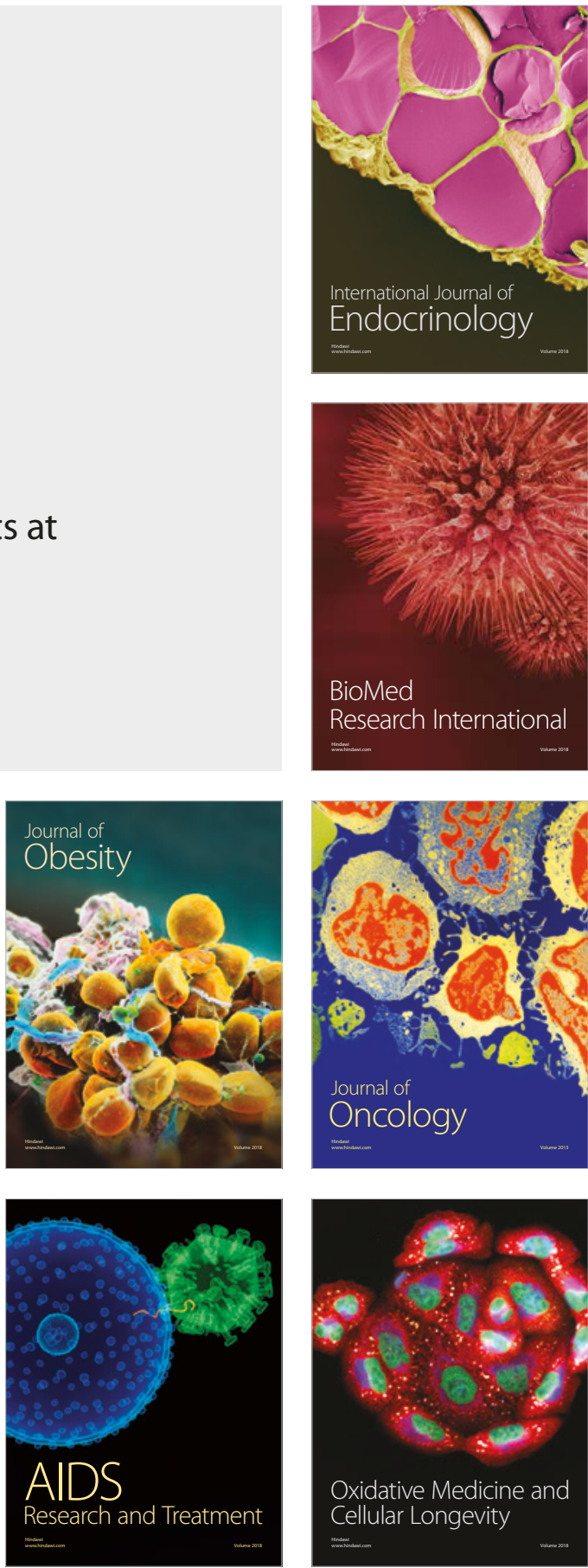